\title{
The involvement of hippocampus and amygdala in schizophrenia
} Asterios Fotiadis*2, Zoe Nassika ${ }^{3}$, Jannis Nimatoudis ${ }^{1}$, Athanasios Karavatos ${ }^{2}$ and George Kaprinis ${ }^{1}$

\author{
Address: ${ }^{1} \mathrm{C}^{\prime}$ Department of Psychiatry, Aristotle University of Thessaloniki, Thessaloniki, Greece, ${ }^{2} \mathrm{~A}^{\prime}$ Department of Psychiatry, Aristotle University \\ of Thessaloniki, Thessaloniki, Greece and ${ }^{3}$ Department of Psychiatry, University of Thessaly, Larissa, Greece \\ * Corresponding author
}

\author{
from International Society on Brain and Behaviour: 3rd International Congress on Brain and Behaviour \\ Thessaloniki, Greece. 28 November - 2 December 2007 \\ Published: 17 April 2008 \\ Annals of General Psychiatry 2008, 7(Suppl I):S34I doi:I0.II86/I744-859X-7-SI-S34I
}

This abstract is available from: http://www.annals-general-psychiatry.com/content/7/SI/S34 I

(c) 2008 Fotiadis et al.; licensee BioMed Central Ltd.

\section{Background}

In recent years, using MRI studies, researchers try to establish valid hypothesis about the role of hippocampus and amygdala in schizophrenia.

\section{Materials and methods}

A critical presentation of meta-analyses and reviews of MRI and post mortem studies in schizophrenic patients that define the role of hippocampus and amygdala. Selective review of MRI and post-mortem studies between 1998-2005, including schizophrenic and bipolar patients, in Medline. Preferential focus on review articles and metaanalyses.

\section{Results}

Volume reductions of 5-8\% are reported in 2/3 of the MRI studies. However these findings are based on many studies including the hippocampus, but only a few studies of amygdala. Therefore amygdala volume reductions can be refuted by unpublished negative studies. The same conclusion can be drawn for postmortem studies; hippocampal abnormalities are more robust than those of amygdala.

Amygdala lesions result in emotional deficits, such as facial affect recognition, that occurs also in schizophrenic patients, especially paranoid, compared to non-paranoid. The volume of amygdala may be inversely correlated with the number of $\mathrm{X}$ chromosomes. Hippocampus lesions can produce symptoms that resemble both the positive and the negative symptoms of schizophrenia. However those volume reductions are not specific findings of schizophrenia, since they are also found in bipolar patients and even in normal elderly persons.

\section{Conclusions}

More studies, with larger sample sizes, are needed to establish if schizophrenic patients possess reduced amygdala volume. We list some of questions remaining to be answered. First the specificity of the abnormality to schizophrenic patients and the possible relationship to specific symptoms or cluster of symptoms. Another open domain for research is whether structural abnormalities in schizophrenia such as in hippocampus or amygdale represent a possible genetic marker leading to a vulnerability disease or is a non specific feature in the cohort of psychiatric patients. 